\title{
Finite zero-simple semigroups over an elementary abelian group
}

\section{C.H. Houghton}

\begin{abstract}
Finite 0-simple semigroups with a Rees representation over an elementary abelian group are considered. The isomorphism classes of such semigroups with class graph $\Gamma$ and coefficient group of order $p^{d}$ are shown to correspond to the orbits under the automorphism group of $\Gamma$ of those subspaces of the circuit space of $\Gamma$ over $z_{p}$ which have codimension at most $d$. A semigroup interpretation of the inclusion relation between subspaces is given and results are obtained on the structure of the automorphism group and the enumeration of the semigroups under consideration.
\end{abstract}

\section{Introduction}

A Rees representation of a completely 0-simple semigroup $S$ was interpreted in [2] as associating with $S$ a graph $\Gamma$, a group $G$, and a cohomology class in $H^{l}(\Gamma, G)$. The isomorphism classes of $\Gamma$ and $G$ are invariants of $S$ and two cohomology classes represent the same semigroup if and only if they are in the same orbit of $H^{\mathcal{I}}(\Gamma, G)$ under the action of the automorphism groups of $\Gamma$ and $G$.

Here we consider the case where $\Gamma$ is finite and $G$ is elementary abelian. The detailed information that we obtain provides a basis for comparison in the general situation. Moreover, this special case occurs frequently among the semigroups of small order; for example, of the 293

Received 3 January 1978. 
0-simple semigroups of order less than or equal to 24 with non-trivial $G, 213$ are associated with an elementary abelian group (see [3]).

If $G$ is elementary abelian of order $p^{d}$, then $G$ is the additive group of a $d$-dimensional vector space over $z_{p}$ and all automorphisms of $G$ are linear. Let $\Gamma$ be any finite graph and let $V$ be a vector space of finite dimension $d$ over a field $F$. Suppose that the circuit space $R$ of $\Gamma$ over $F$ has dimension $e$. We show that the set of GL(V)orbits of $H^{l}(\Gamma, V)$ is bijective with the set of subspaces of the circuit space having dimension at least $e-d$. Thus the orbits of $H^{1}(\Gamma, V)$ under $\operatorname{aut}(\Gamma) \times G L(V)$ may be identified with the orbits under aut $(\Gamma)$ of the appropriate subspaces of $R$. Now let $\Gamma$ be a directed graph with disjoint source and target sets and let $V$ be the vector space

corresponding to the elementary abelian group $G$ of order $p^{d}$. Then each isomorphism class of semigroups associated with $\Gamma$ and $G$ will correspond to an orbit of subspaces of $R$ under the action of aut( $\Gamma)$. In particular, as $d$ increases, with $\Gamma$ and $p$ fixed, the number of associated semigroups becomes constant.

The subspaces of $R$ form a lattice and we show that the inclusion relation corresponds to the existence of idempotent-separating homomorphisms between associated semigroups. We describe the automorphism groups of the semigroups under consideration and we show that a simple semigroup with elementary abelian coefficient group rarely admits the action of the whole of aut $(\Gamma)$. Finally, we apply the initial results to investigate the number of isomorphism classes of semigroups associated with certain graphs $\Gamma$.

We begin with a summary of the relevant graph theory; further details may be found in [1].

\section{Cohomology of graphs}

Suppose that $\Gamma$ is a finite directed graph and that $V$ is a finite dimensional vector space over the field $F$. A map from the edge set of $\Gamma$ to $V$ is called a cochain (or chain, since $\Gamma$ is finite). Such maps form a vector space $C(\Gamma, V)$. Given a map $t$ from the vertex set of $\Gamma$ to $V$, we can define a corresponding cochain by sending an edge with source $x$ 
and target $y$ to $y t-x t$. Such cochains are called coboundaries and they form a subspace $B(\Gamma, V)$ of $C(\Gamma, V)$. The quotient space $C(\Gamma, V) / B(\Gamma, V)$ is the first cohomology group $H^{l}(\Gamma, V)$ of $\Gamma$ with coefficients in $V$.

If $X$ is the vertex set of $\Gamma$, then the edge set of $\Gamma$ can be identified with a subset $E$ of $X \times X$. Given a group $A$ of automorphisms of $\Gamma$, we can define an action of $A$ on $C(\Gamma, V)$. For $\theta \in A, q \in C(\Gamma, V)$, and $(x, y) \in E$, we put

$$
(x, y)(q \theta)=\left(x \theta^{-1}, y \theta^{-1}\right) q \text {. }
$$

Then $A$ leaves $B(\Gamma, V)$ invariant and hence $B^{\mathcal{I}}(\Gamma, V)$ is an $A$-group. In a similar way, there is an action of the general linear group $L=G L(V)$ on $C(\Gamma, V)$ and on $H^{l}(\Gamma, V)$. For $q \in C(\Gamma, V), \alpha \in L$, and $(x, y) \in E$, we put $(x, y)(q \alpha)=((x, y) q) \alpha$. The actions of $A$ and $L$ commute and so $H^{1}(\Gamma, V)$ is an $(A \times L)$-group.

Taking $V=F$, we can associate with each $c \in C(\Gamma, F)$, a map $c \delta$ from $X$ to $F$ by letting $x(c \delta)$ be the sum of all $(y, x) c$ and $-(x, y) c$ as $y$ ranges over the vertices adjacent to $x$. The kernel $R$ of $\delta$ is the circuit space of $\Gamma$ over $F$. It is spanned by the elements of $C(\Gamma, F)$ obtained from the circuits in $\Gamma$, where an edge sequence is associated with the corresponding signed sum of edges. For any $V, a$ cochain $q_{I} \in C(\Gamma, V)$ induces a linear transformation from $C(\Gamma, F)$ to $V$, whose restriction to $R$ we denote by $q$. A standard result of cohomology $[5, p .165]$ asserts that the map $q_{1} \rightarrow q$ induces an isomorphism between $H^{1}(\Gamma, V)$ and $\operatorname{hom}_{F}(R, V)$. This can be seen by first choosing a maximal forest $T$ in $\Gamma$. Then each class in $H^{l}(\Gamma, V)$ contains a unique element trivial on $T$. But the circuits in $\Gamma$ with just one edge not in $T$ form a basis for $R$ and the result follows.

We now investigate the action of $L=G L(V)$ on $\operatorname{hom}_{F}(R, V)$. If $K(q)$ denotes the kernel of $q \in \operatorname{hom}_{F}(R, V)$ and if $\alpha \in L$, then $K(q)=K(q \alpha)$. Conversely, suppose $K(q)=K(p)$, with $p \in \operatorname{hom}_{F}(R, V)$. 
Then defining $(r q) \alpha=r p$ gives a well-defined isomorphism from the image of $q$ to the image of $p$ and this extends to an automorphism of $V$. If $\operatorname{dim}(R)=e$ and $\operatorname{dim}(V)=d$, then $\operatorname{dim}(K(q)) \geq e-d$. Now any subspace of $R$ of dimension at least $e-d$ is the kernel of some $q \in \operatorname{hom}_{F}(R, V)$. Thus the orbits of $H^{1}(\Gamma, V)$ under the action of $L$ are in one-to-one correspondence with the subspaces of $R$ having codimension at most $d$. Then the action of $A \times L$ on $H^{1}(\Gamma, V)$ will induce an action of $A$ on these subspaces. If $\theta \in A$, then $r(q \theta)=\left(r \theta^{-1}\right) q$, for $r \in R$, and so $r \in K(q \theta)$ if and only if $r \in K(q) \theta$. Thus the action of $A$ on the subspaces is induced by the natural action of $A$ on $R$. We summarise these results.

THEOREM 1. Suppose that $R$ is the circuit space of a finite graph $\Gamma$ over the field $F$. If $V$ is a finite dimensional vector space over $F$ and $A$ is a group of automorphisms of $\Gamma$, then there is a bijection between the $(A \times \mathrm{GL}(V))$-oxbits of $H^{\mathcal{l}}(\Gamma, V)$ and the A-orbits of subspaces of $R$ having codimension at most equal to the dimension of $V$.

We now apply these results to semigroups, using the terminology of [4].

\section{Application to semigroups}

Rees [6] showed that a semigroup is completely 0-simple if and only if it is isomorphic to a regular Rees semigroup $M=M^{0}(G ; I, \Lambda ; q)$. Such a semigroup is determined by a group $G$, sets $I$ and $\Lambda$, and a map $q$ from $\Lambda \times I$ to $G \cup\{0\}$ such that the support of $q$ projects onto $\Lambda$ and $I$. The class graph $\Gamma$ of $M$ is the directed bipartite graph whose edges are all $(\lambda, i)$ such that $(\lambda, i) q \neq 0$; the condition on $q$ implies that the vertex set of $\Gamma$ is $\Lambda \cup I$. Then $q$ defines a cochain and hence a cohomology class of $\Gamma$. It is shown in [2] that the set of isomorphism classes of completely 0-simple semigroups associated with fixed $\Gamma$ and $G$ is bijective with the set of orbits of $H^{\mathcal{l}}(\Gamma, G)$ under the action of aut $(\Gamma) \times \operatorname{aut}(G)$. If $G$ is elementary abelian of order $p^{d}$, then $\operatorname{aut}(G)=\operatorname{aut}_{F}(V)$, where $F=Z_{p}$ and $V$ is the $F$-space corresponding to $G$. Each finite 0 -simple semigroup is completely 0 -simple and so we have the following consequence of Theorem 1. 
THEOREM 2. Suppose that $\Gamma$ is a directed graph with a finite set $\Lambda \cup I$ of vertices such that each edge has source in $\Lambda$ and target in $I$ and no vertices are isolated. Let $G$ be an elementary abelian group of order $p^{d}$ and let $R$ be the circuit space of $\Gamma$ over $z_{p}$. Then the set of isomorphism classes of finite 0-simple semigroups with class graph $\Gamma$ and coefficient group $G$ is bijective with the aut $(\Gamma)$-orbits of subspaces of $R$ having codimension at most $d$.

COROLLARY 3. The number of o-simple semigroups with fixed finite graph $\Gamma$ and elementary abelion coefficient group of order $p^{d}$, becomes constant as d increases.

We note that if $M=M^{0}(G ; I, \Lambda ; q)$ has class graph $\Gamma$, then the subspace $K$ associated with $M$ by Theorems 1 and 2 is the kernel of the map from $R$ to $V$ induced by $q$. The isomorphism class of $M$ is determined by the graph $\Gamma$, the group $G$, and the subspace $K$.

A finite 0-simple semigroup is orthodox if and only if it corresponds to the trivial cohomology class of a graph $\Gamma$ which is a union of disjoint complete bipartite graphs [2]. Suppose $K$ is a subspace of $R$ corresponding to a 0-simple semigroup $M$ with graph $\Gamma$ of this type and with $G$ elementary abelian. Then the codimension of $K$ represents the degree of unorthodoxy of $M$. Similarly, for general $\Gamma$, the codimension of $K$ measures the obstruction to weak orthodoxy, where a semigroup is said to be weak orthodox if each $H$-class contains at most one non-zero product of idempotents [2].

The subspaces of $R$ form a lattice and our next result gives an interpretation, in terms of semigroups, of the sublattice above a particular subspace of $R$. Here the graph $\Gamma$ is fixed but $G$ may vary.

THEOREM 4. Let the semigroup $M$ correspond to a subspace $K$ of $R$ together with the additive group of the vector space $V$ of dimension $d$ over $Z_{p}$. Suppose that $J$ is a subspace of $R$ containing $K$ and that $N$ is a semigroup associated with $J$ and with a vector space $W$ over $Z_{p}$ such that

$$
\operatorname{dim}(R)-\operatorname{dim}(J) \leq \operatorname{dim}(W) \leq \operatorname{dim}(V)-\operatorname{dim}(J)+\operatorname{dim}(K) .
$$


Then $N$ is the image of an idempotent separating homomorphism of $M$ and each such image of $M$ corresponds to a subspace $J$ and space $W$ satisfying the given conditions.

Proof. Let $M=M^{0}(V ; I, \Lambda ; q)$, where $K$ is the kernel of the map from $R$ to $V$ induced by $q$. Then there is a natural epimorphism from $R q$ to $R / J$ which can be extended to a homomorphism $\omega$ from $V$ onto the space $W$. From [2, Theorem 3.6], the map $\omega$, in conjunction with the identity map on $\Gamma$, defines an idempotent separating homomorphism from $M$ onto the semigroup $M=M^{0}(V \omega ; I, \Lambda ; q \omega)$. The corresponding subspace of $R$ is the kernel $J$ of the map induced by $q w$ on $R$.

Conversely, from [2, Theorem 3.6], an image of $M$ under an idempotent separating homomorphism will be isomorphic to a semigroup $N$ defined by $q \omega \in \operatorname{hom}_{F}(R, W)$, where $\omega$ is an epimorphism from $V$ to $W$. Then the kernel $J$ of $q w$ contains $K$ and $\operatorname{dim}(W)$ satisfies the given inequality.

A normal series for the automorphism group of a completely 0-simple semigroup was given in [2]. We interpret this result in our special situation.

THEOREM 5. Let $M$ be the finite regular semigroup $M^{0}(V ; I, \Lambda ; q)$, with $V$ elementary abelian. Then $L=$ aut $(M)$ has a normal series $L \geq L_{1} \geq L_{2} \geq L_{3} \geq 1$, where

(i) $L / L_{1}$ is isomorphic to the subgroup of aut( $\left.\Gamma\right)$ fixing the kernel of the map from $R$ to $V$ induced by $q$,

(ii) $L_{1} / L_{2}$ is isomorphic to $\mathrm{GL}(W)$, where $W$ is an F-space of dimension $\operatorname{dim}(V)-\operatorname{dim}(R q)$,

(iii) $L_{2} / L_{3}$ is isomorphic to the $\operatorname{direct}$ sum of $\operatorname{dim}(V)-\operatorname{dim}(R q)$ copies of $R q$,

(iv) $L_{3}$ is the direct sum of $c-1$ copies of $z_{p}$, where $c$ is the number of components of $\Gamma$.

Proof. From Theorems 3.3 and 3.5 of [2], $L / L_{1}$ is 1somorphic to the 
image in aut $(\Gamma)$ of the $(\operatorname{aut}(\Gamma) \times \mathrm{GL}(V))$-stabiliser of $q$ and so (i) follows from Theorem 2. The description of $L_{3}$ is given in [2] and $L_{1} / L_{3}$ is shown to be the subgroup of $G L(V)$ consisting of those maps fixing $R q$ elementwise. The result follows by considering the effect of such a map on a basis of $V$ extending a basis of $R q$.

If the kernel of $q$ is the maximum or minimum element of the lattice of subspaces of $R$, then $L / L_{1}=\operatorname{aut}(\Gamma)$. We shall consider a situation in which the semigroups corresponding to these subspaces are the only ones admitting the whole automorphism group of $\Gamma$. This is equivalent to the irreducibility of $R$ as an aut( $\Gamma)$-space.

A finite 0-simple semigroup is the union of $\{0\}$ and a simple semigroup if and only if $\Gamma$ is a complete bipartite graph $B(m, n)$, with edges directed from left to right [2]. The automorphism group of $\Gamma$ is then $S_{m} \times S_{n}$. We shall investigate the action of this group on the circuit space of $\Gamma$.

There is a natural representation of $S_{n}$ on $F^{n}$ given by permuting a basis $\left\{e_{1}, \ldots, e_{n}\right\}$ according to the action of $S_{n}$. This induces a representation $\rho_{n}$ on the subspace $J_{n}$ of vectors $\sum \lambda_{i} e_{i}$ with $\sum \lambda_{i}=0$. Let $J_{1}$ be the cyclic module generated by $j=\sum \lambda_{i} e_{i} \in J$ under the action of $S_{n}$. If $\lambda_{h} \neq \lambda_{i}$, then $j-j(h i)=\left(\lambda_{h}-\lambda_{i}\right)\left(e_{h}-e_{i}\right)$ and so $J_{1}=J$. Thus $J$ is irreducible, unless $\sum \lambda e_{i} \epsilon J$, for some $\lambda \neq 0$. This occurs if and only if $p$ divides $n$.

THEOREM 6. The representation of $S_{m} \times S_{n}$ on the circuit space of $B(m, n)$ over the field $F$ is $\rho_{m} \otimes \rho_{n}$ and is irreducible unless the characteristic $p$ of $F$ divides $m$ or $n$.

Proof. The natural permutation representation of $S_{m} \times S_{n}$ on $\{1, \ldots, m\} \times\{1, \ldots, n\}$ induces a linear representation on $F^{m} \otimes F^{n}$, where bases $\left\{e_{1}, \ldots, e_{m}\right\}$ and $\left\{f_{1}, \ldots, f_{n}\right\}$ are permuted according to their suffices. Now $F^{m} \otimes E^{n}$ is isomorphic to the space $C(\Gamma, F)$ and the 
action of $S_{m} \times S_{n}$ on $R$ is induced by its action on $C(\Gamma, F)$. The element $\left\{\sum \lambda_{i} e_{i}\right\} \otimes\left\{\sum \mu_{j} f_{j}\right\}=\sum \lambda_{i} \mu_{j}\left(e_{i} \otimes f_{j}\right)$ corresponds to an element of $R$ if and only if $0=-\sum \lambda_{i}\left(\sum \mu_{j}\right) e_{i}+\sum \mu_{j}\left(\sum \lambda_{i}\right) f_{j}$; that is, $\sum \lambda_{i}=\sum \mu_{j}=0$. Thus $R$ contains the space $J_{m} \otimes J_{n}$. But $R$ has dimension $m n-m-n+1=(m-1)(n-1)$ and so $R=J_{m} \otimes J_{n}$; hence the representation of $S_{m} \times S_{n}$ on $R$ is $\rho_{m} \otimes \rho_{n}$. If $p$ divides neither $m$ nor $n$, then $\rho_{m}$ and $\rho_{n}$ are irreducible, so $\rho_{m} \otimes \rho_{n}$ is irreducible.

COROLLARY 7. Suppose that $p$ divides neither $m$ nor $n$, and that $M$ is a simple semigroup with class graph $B(m, n)$ and elementary abelian coefficient group of order $p^{d}$. Then $M$ admits the full automorphism group $S_{m} \times S_{n}$ of the directed graph $B(m, n)$ if and only if it corresponds to the maximum or minimum element of the Zattice of subspaces of the circuit space of $B(m, n)$ over $Z_{p}$.

Theorem 2 gives a method of counting the semigroups associated with a graph $\Gamma$ and an elementary abelian group of order $p^{d}$. We consider some special cases.

Let $e$ be the dimension of the circuit space $R$. If $\Gamma$ has trivial automorphism group, then the isomorphism classes of semigroups simply correspond to the subspaces of codimension at most $d$ of the vector space of dimension $e$ over $z_{p}$. Now suppose that $\operatorname{aut}(\Gamma)$ is unrestricted. We have $e=0$ if and only if $\Gamma$ is a forest and then there is just one isomorphism class of semigroups. If $e=1$, then $R$ has two subspaces, both fixed by aut( $\Gamma)$, so there are two semigroups. For $e>1$, it is convenient to treat $R$ as a projective space $P R$. In the case $e=2, P R$ is a projective line and, for given $\Gamma$, the action of $\operatorname{aut}(\Gamma)$ can usually be determined without difficulty. As an

illustration of the case $e=3$, let $\Gamma$ be the graph $B(3,3)$ with one edge removed. The automorphism group of the directed graph $\Gamma$ is $C_{2} \times C_{2}$ and, for $p=2, P R$ is the projective plane with seven points. The action of aut $(\Gamma)$ can be shown to fix one point and the three lines 
through that point, so, for $d \geq 3$, the number of semigroups is $1+4+4+1=10$, with an appropriate reduction if $d<3$. For $p>2$, one can apply Burnside's Lemma, which states that the number of orbits is $|\operatorname{aut}(\Gamma)|^{-1} \sum F(\theta)$, where $F(\theta)$ is the number of subspaces fixed by $\theta$, and $\theta$ runs through aut( $\Gamma)$. Each non-trivial element of our automorphism group fixes a point and a disjoint line pointwise and thus, for $d \geq 3$, the number of orbits is

$$
\frac{1}{4}\left(2\left(1+p^{2}+p+1\right)+(3 \times 2)(1+1+p+1)\right)=\frac{1}{2}\left(p^{2}+4 p+11\right) .
$$

For $d<3$, one omits the fixed subspaces of dimension less than $3-d$.

The last example illustrates some general principles involved in counting the semigroups associated with a particular graph $\Gamma$. To apply Burnside's Lemma, one must find the subspaces fixed by $\theta \in$ aut( $\Gamma$ ). If $p$ does not divide the order of $\theta$, then $R$ is completely reducible as a $Z_{p}(\theta)$-module and each fixed subspace is a direct sum of irreducible subspaces. Now $\theta$ is an element $(\alpha, \beta)$ of $S_{m} \times S_{n}$, where $m$ and $n$ are the orders of the source and target sets of $\Gamma$. The eigenvalues of $\alpha$ acting on $J_{m}$ and $\beta$ acting on $J_{n}$ are roots of unity in a suitable splitting field and the eigenvalues of $\theta$ acting on $J_{m} \otimes J_{n}$ are all the products of an eigenvalue of $\alpha$ and of $\beta$. One can thus determine the decomposition of $J_{m} \otimes J_{n}$ as a direct sum of irreducible $z_{p}\langle\theta\rangle$-modules and hence the decomposition of its submodule $R$. This procedure is not available when $p$ divides the order of $\theta$, in which case one works directly in terms of $R$.

Using these methods, one can derive formulae for the number of isomorphism classes of semigroups associated with elementary abelian groups and various classes of graphs. This complements the crude counting procedure of [3], which takes no account of the structure of the class graph. As a typical example, let $c\left(p^{d}\right)$ be the number of simple semigroups with three $L$-classes, two R-classes, and elementary abelian coefficient group of order $p^{d}$. If $d>2$, then $c\left(2^{d}\right)=2, c\left(3^{d}\right)=4$, and for $p>3, c\left(p^{d}\right)=3+(p+1) / 6$ if $p \neq 1(\bmod 3)$, 
$c\left(p^{d}\right)=4+(p-1) / 6$ if $p \equiv 1(\bmod 3)$.

\section{References}

[1] Norman Biggs, Algebraic graph theory (Cambridge Tracts in Mathematics, 67. Cambridge University Press, Cambridge, 1974).

[2] C.H.Houghton, "Completely 0-simple semigroups and their associated graphs and groups", Semigroup Fomm 14 (1977), 41-67.

[3] C.H. Houghton, "Counting completely O-simple and completely simple semigroups", Proc. Roy. Soc. Edinburgh Ser. A (to appear).

[4] J.M. Howie, Introduction to semigroup theory (Academic Press [Harcourt Brace Jovanovich], London, New York, San Francisco, 1976).

[5] C.R.F. Maunder, Algebraic topology (Van Nostrand, London, New York, Cincinnati, Toronto, Melbourne, 1970).

[6] D. Rees, "On semi-groups", Proc. Combridge Philos. Soc. 36 (1940), $387-400$.

Department of Pure Mathematics,

University College,

Cardiff,

Wales. 